Meta

Journal des traducteurs

Translators' Journal

\title{
Une méthodologie de la traduction médicale
}

\section{Amal Jammal}

Volume 44, numéro 2, juin 1999

URI : https://id.erudit.org/iderudit/003249ar

DOI : https://doi.org/10.7202/003249ar

Aller au sommaire du numéro

Éditeur(s)

Les Presses de l'Université de Montréal

ISSN

0026-0452 (imprimé)

1492-1421 (numérique)

Découvrir la revue

Citer cet article

Jammal, A. (1999). Une méthodologie de la traduction médicale. Meta, 44(2), 217-237. https://doi.org/10.7202/003249ar

\section{Résumé de l'article}

Cet article fait un tour d'horizon des difficultés les plus courantes en traduction médicale. L'auteur propose une méthodologie propre à ce domaine, permettant au traducteur de trouver des solutions et d'éviter les nombreux pi'ges de cette langue de spécialité. d'utilisation que vous pouvez consulter en ligne.

https://apropos.erudit.org/fr/usagers/politique-dutilisation/ 


\title{
Une méthodologie de la traduction médicale
}

\author{
amal jammal \\ Université de M ontréal, M ontréal, Canada
}

\begin{abstract}
RÉSUMÉ
Cet article fait un tour d'horizon des difficultés les plus courantes en traduction médicale. L'auteur propose une méthodologie propre à ce domaine, permettant au traducteur de trouver des solutions et d'éviter les nombreux pièges de cette langue de spécialité.
\end{abstract}

\section{ABSTRACT}

This article provides an overview of the most recurrent problems in medical translation. The author propose a method specifically adapted to this specialty to enable the translator to come up with solutions and avoid the numerous pitfalls so common to this field.

Pourquoi parler d'une méthodologie particulière à la traduction médicale? En quoi la traduction dans ce domaine diffèret-elle de celle qui se pratique dans d'autres domaines, comme l'économique, le technique ou le juridique par exemple? N e s'agitil pas essentiellement, comme pour toute traduction, d'une opération de décodage, suivie d'une opération de transcodage?

Pour notre part, nous croyons que la traduction médicale diffère des autres champs de traduction sous certains aspects.

Ainsi, prenons la phase de décodage. II est heureusement devenu un truisme de nos jours de dire que l'on ne peut traduire que ce que l'on comprend bien. Pour décoder, il faut donc bien comprendre. Or il nous semble que, de tous les domaines, le domaine médical est peut-être celui qui est le plus difficile à appréhender. On conviendra, par exemple, qu'il est plus facile de comprendre les tenants et aboutissants du traité de M aastricht, ou la relation existant entre l'inflation et le chômage, que ce qui se passe au cours de la biosynthèse du cholestérol et des troubles qui peuvent causer l'hypercholestérolémie; ou encore pourquoi, jusqu'à présent, nous restons impuissants devant le virus de l'immunodéficience humaine. De même, il suffit de se rappeler que la plus petite cellule du corps humain est plus complexe dans sa structure et son fonctionnement que le plus complexe des moteurs ou des ordinateurs, ou que la législation la plus compliquée; rappelons encore que ceux-ci sont nés du cerveau humain, alors que ledit cerveau reste encore, pour une grande part, terra incognita.

On pourrait donc déduire que, pour qui n'est pas médecin, les textes médicaux ne se laissent pas facilement décoder. Faut-il conclure pour autant que seuls les médecins sont habilités à faire de la traduction médicale? Non et, de toute évidence, ce n'est pas le cas. En effet, rares sont les médecins qui consentent à troquer leur stéthoscope contre la plume et le dictionnaire. Et s'ils le faisaient, il leur faudrait, en sus, apprendre à éviter les nombreux pièges que comporte l'opération traduisante (interférences entre langue de départ et langue d'arrivée, difficultés syntaxiques inhérentes aux différences structurelles des deux langues, etc.). À défaut donc du traducteur 
idéal - qui serait un médecin ayant reçu une formation en traduction - il existe des traducteurs médicaux qui ne sont pas des disciples d'Esculape et qui néanmoins font très honorablement leur métier. Comment y parviennent-ils?

C'est là que nous proposons une méthodologie particulière, qui s'intéressera successivement à la phase du décodage et à celle du transcodage.

\section{A. LA PHASE DU DÉCODAGE}

Elle implique une démarche documentaire méthodique, soumise à des contraintes qui sont, en apparence du moins, assez contradictoires, une prise de conscience de certains aspects particuliers qui pourraient faire obstacle à la compréhension du texte et enfin une recherche terminologique systématique.

\section{La démarche documentaire}

Elle doit être méthodique et ordonnée mais, assez paradoxalement, rigoureusement limitée et soumise à une autodiscipline intellectuelle stricte. Nous nous expliquons.

a) Une démarche méthodique et ordonnée

Nous parlions plus haut de la nécessité de comprendre le texte avant de le traduire. II nous faut mentionner, à ce stade, la distinction cruciale que fait Schumacher (1973: 310) entre compréhension et connaissance. II découle de cette distinction que, dans le processus de la cognition, la compréhension est l'échelon le plus élémentaire si la connaissance en est l'étape ultime. Dans le domaine médical, où la compréhension pour qui n'est pas médecin peut paraître une réelle gageure, nous prétendons qu'il est possible de comprendre un sujet à l'aide d'une documentation efficace. Cela ne signifie pas toutefois qu'on connaîtra ce sujet. On peut comprendre le processus pathologique qui sous-tend l'inflammation articulaire par exemple, on ne devient pas pour autant un rhumatologue. Nécessité donc, avant de traduire, de se documenter à fond. Mais par où commencer et quoi chercher? Là, nous proposons au traducteur un itinéraire logique. À la lecture du document à traduire, un document qui, dans la plupart des cas, traitera d'une maladie donnée ou encore d'un médicament proposé pour le traitement d'une maladie, il lui faut tout d'abord déterminer quel est l'appareil, le système, l'organe ou les tissus que la maladie attaque. II lui faut ensuite se documenter sur la structure de cet appareil ou de cet organe, donc sur son anatomie - ou sur son histologie dans le cas des tissus - , puis sur son fonctionnement, donc sur sa physiologie, ensuite sur la maladie elle-même (son étiologie, son évolution, ses signes et symptômes, etc.) et enfin sur le médicament qui est censé la traiter (la classe à laquelle il appartient, sa composition, son mode d'action, son effet thérapeutique, ses effets indésirables, etc.).

Voici donc ce que serait l'ordre séquentiel d'une telle démarche documentaire:

- anatomie;

- physiologie;

- pathologie;

- pharmacologie.

Cette méthode est en fait celle que nous avons suivie dans la rédaction de L'hypertension artérielle (Jammal et Dussault 1983), un ouvrage conçu essentiellement 
pour les étudiants en traduction médicale, afin d'illustrer la démarche documentaire proposée. Nous y avons suivi l'ordre séquentiel cité plus haut; I'ouvrage comprend donc, outre des lexiques et un glossaire qui pourraient aider à la phase de recherche terminologique, l'information nécessaire à la «compréhension » de I'hypertension artérielle et de l'arsenal thérapeutique qu'on lui oppose.

Prenons, à titre d'exemple, le cas d'un tiré à part traitant des inhibiteurs de l'enzyme de conversion del'angiotensine, une classe d'antihypertenseurs qui a actuellement le vent dans les voiles. Pour qui n'a pas suivi la démarche documentaire que nous venons de décrire - et évidemment s'il s'agit d'un profane - le texte pourrait paraître totalement abscons. Par contre, pour qui aura compris l'anatomie et la physiologie du rein, le mécanisme de régulation rénale en cause dans la tension artérielle et comment ce mécanisme peut gripper, produire en excès une certaine substance et mener à I'hypertension, l'article ne posera guère de problème de décodage.

Autre exemple: impossible de traduire un texte scientifique sur le sida sans savoir quelles sont les composantes du système immunitaire, comment celui-ci fonctionne face à l'agresseur, ce qu'est un «rétrovirus» et comment celui-ci parvient à brouiller les cartes et à vaincre les défenses naturelles de l'organisme.

C'est dire qu'il s'agit avant tout de résister au chant des sirènes - en l'occurrence à la tentation de sauter des étapes, en misant sur ses propres capacités déductives, intuitives ou divinatoires - et de s'interdire de traduire un texte médical, si simple qu'il puisse paraître à première vue, avant de s'assurer que l'on a bien compris ce dont il s'agit. La démarche peut paraître longue et chronophage, elle n'en est pas moins cruciale si l'on veut éviter les faux-sens ou, pis encore, les non-sens... et elle n'est jamais lassante. De plus, elle a d'autres avantages non négligeables: à lire sur le sujet, on acquiert sans douleur et par un phénomène d'osmose, le vocabulaire que nous appellerions «périphérique», c'est-à-dire les cooccurrents de certains termes, des tournures de phrase particulières, bref toute la phraséologie propre au sujet.

N écessité donc d'une démarche documentaire méthodique, qu'il faut néanmoins baliser par :

\section{b) Une discipline intellectuelle rigoureuse}

II ne suffit pas en effet que la démarche documentaire soit méthodique, il faut aussi qu'elle soit soumise à une discipline de l'esprit car, aussi étonnant que cela puisse paraître, le plus difficile dans ce métier, ce n'est pas de devoir se documenter, mais d'avoir assez de volonté pour cesser de le faire. La tentation est grande en effet d'en savoir toujours plus, d'aller toujours plus loin. Après tout, qu'est-ce qui peut nous intéresser plus que notre corps et ce qui risque de l'affecter? Or, il nous faut brider cette curiosité naturelle car, si elle ne risque pas de nous changer en statue de sel comme elle l'a fait pour la femme de Loth, elle peut avoir deux effets pervers:

1. elle fait perdre du temps, denrée rare et précieuse pour le traducteur à qui, comme on

le sait, le travail est toujours réclamé pour la veille; et

2. elle rend hypocondriaque: à trop se pencher sur les signes et symptômes des maladies, le traducteur finit toujours par découvrir qu'il en souffre, et au stade aigu encore. II risque ainsi de se retrouver atteint d'une kyrielle de maladies dont il peut se demander comment elles n'ont pas encore eu raison de lui.

Passée donc l'étape de la documentation, nous présumons que le traducteur a compris globalement ce dont il s'agit. Nous disons bien globalement, parce que la 
phase de décodage peut comporter certains pièges sémantiques particuliers au domaine médical, que le traducteur doit connaître s'il veut les éviter : ceux qu'entraînent les interférences entre la langue de départ et la langue d'arrivée'; ceux qui sont inhérents à la structure syntaxique de l'anglais; ceux que provoque parfois une nébulosité terminologique dans le texte de départ.

\section{Le repérage des pièges sémantiques}

a) Les «faux amis» sémantiques

Nous n'avons nullement la prétention de dresser ici une liste exhaustive des anglicismes particuliers à la langue médicale. N ous signalerons seulement une catégorie plus pernicieuse, celle qui contient les anglicismes les plus sournois, ceux qui ne sont visibles qu'à l'œil averti : les faux amis sémantiques. Comme on le sait, ce sont des termes qui, en anglais et en français, ont une grande similitude de forme, mais une nette divergence de sens. En voici quelques-uns:

- abnormality: n'est pas *anormalité en français (puisque ce terme semble ne s'appliquer qu'à tout écart par rapport à une norme établie par le cerveau humain), mais bien anomalie (qui qualifie une déviation biologique).

- aggressive (comme dans aggressive treatment) : on s'en doute, ce terme ne se rend pas par *agressif en français lorsqu'il s'agit de qualifier un traitement, l'objectif n'étant pas d'achever le malade. Celui-ci en effet, déjà agressé par la maladie, n'a pas besoin de l'être aussi par le traitement. C'est pourquoi on parlera plutôt d'un traitement énergique.

- alternative: en français, une alternative est le choix entre deux solutions. En anglais, par contre, le mot désigne l'autre solution. Autrement dit, lorsque l'anglais dit: «The alternative choice would be...», le français ne peut traduire par: «*Le choix alternatif serait... », mais plutôt par : «L'autre choix serait... ».

- $\quad$ approach : ce terme traduit par approche, dans le sens de démarche, provoquait naguère l'ire des puristes. Pourtant, il a désormais son entrée dans au moins un dictionnaire respectable (Le petit Robert 1994: 107), même si un autre dictionnaire non moins respectable continue à le faire suivre de la mention «calque de l'anglais, emploi critiqué» (Le petit Larousse illustré 1998: 77-78). Le terme anglais pour sa part a, comme le souligne Van Hoof (1986: 98), deux acceptions, l'une générale et l'autre plus technique. A notre avis, en étendant ainsi son champ sémantique, du moins dans le domaine médical, il a perdu quelque peu de sa concision, car il a fini par renvoyer à des référents aussi disparates que démarche, notion abstraite, et voie d'abord, notion éminemment concrète. Ainsi, on peut avoir :

The physician's approach was very conservative soit, en français:

\section{La démarche du médecin était très prudente} mais on peut avoir aussi :

Hysterectomy can be performed by the abdominal or the vaginal approach soit, en français:

\section{L'hystérectomie peut être effectuée par voie abdominale ou par voie vaginale.}

Si donc le terme «approche» au sens de «démarche» est de plus en plus accepté dans le langage courant, dans le langage médical, au sens de «voie d'abord», il est à éviter. 
- clearance: ce terme est un cas particulier. Longtemps, certains incorruptibles de la langue ont maintenu qu'il fallait le rendre par épuration. Actuellement, on parle de clairance ou même de clearance, lui donnant ainsi, mal gré son apparence, la nationalité française.

- $\quad$ condition : ne se traduit pas par *condition en français, mais plutôt par état. Exemple: «L'état - et non la condition - de ce malade est satisfaisant. »

- $\quad$ conservative (comme dans conservative treatment) : n'est pas *conservateur en français (même si l'objectif est de conserver le malade), mais plutôt modéré ou prudent.

- $\quad$ conventional : ne se traduit pas par *conventionnel, mais plutôt par traditionnel, courant, classique. Exemple: «The conventional treatment includes diuretics» se traduirait par : «Le traitement classique comprend des diurétiques. »

- $\quad$ to control : il s'agit d'un verbe qui peut avoir plus d'un sens en anglais et qui signifie, en français, vérifier mais aussi, selon une acception récemment admise par les dictionnaires, prendre le dessus, maîtriser. Dans la langue générale ou dans d'autres langues de spécialité, cette polysémie et l'ambiguïté qui en résulte peuvent ne pas prêter à conséquence. $M$ ais dans la langue médicale, elle est parfois gênante. Lorsqu'on précise que le médecin must control a patient's hypertension, dit-on qu'il lui faut abaisser les chiffres tensionnels de ce malade ou qu'il doit le voir à intervalles réguliers pour vérifier sa tension artérielle? Fort heureusement, ce verbe pluriréférentiel anglais a, en français, plusieurs équivalents monoréférentiels possibles qui diffèrent selon le cooccurrent auquel ils sont accolés. Ainsi on peut avoir pour to control anxiety: calmer l'anxiété; to control diabetes: équilibrer le diabète. On écrira aussi assécher un œè̀me, corriger des effets indésirables; dans certains cas, lutter contre une maladie, abaisser ou réduire des chiffres tensionnels élevés, ou les stabiliser s'ils ont atteint le niveau désiré, etc.

- course: comme le signale Van Hoof (1970: 103), ce terme peut avoir deux sens: il peut signifier évolution ; exemple: «The course of brucellosis is usually protracted »: «L'évolution de la brucellose est habituellement longue»; ou il peut signifier aussi série ( $d$ 'injections par exemple) : «There was no response to a course of penicillin injections», qui se rendrait alors par : "La série d'injections de pénicilline demeura sans effet. »

- damage: I'anglais traite le corps comme une machine ou un moteur. Lorsqu'il y a dysfonctionnement ou agression de l'organisme, il parlera de damage. Le français rend cette notion par altération ou trouble ou encore lésion.

- defect: même réflexion pour ce terme, utilisé généralement pour toute anomalie. Le français utilise le mot spécifique, plus descriptif de la réalité et qui change selon le cooccurrent. Exemple: defect of visual field se rend par lacune du champ visuel, ou encore valvular defect par anomalie, affection, lésion valvulaire (Van Hoof 1970: 99).

- development (dans le contexte de l'industrie pharmaceutique) : ne se traduit pas par *développement s'il s'agit d'un produit, mais par la mise au point de ce produit. S'il s'agit de symptômes ou de maladies, on parlera de leur manifestation, de leur survenue, de leur apparition.

- discharge: terme anglais qui peut avoir deux sens, l'un général, l'autre spécialisé. Ainsi, dans: «At his discharge the ECG was normal », le mot discharge devient en français «à sa sortie» ou «à son congé de l'hôpital » (I'ECG était normal). M ais ce terme peut avoir aussi un deuxième sens, celui d'écoulement. Ainsi : «In the second patient, there was no profuse nasal discharge» se traduirait en français par : «Chez le deuxième malade, il n'y a pas eu d'écoulement nasal abondant. »

- $\quad$ disposable: surtout ne se traduit pas par *disposable mais, selon les circonstances, par jetable, à usage unique, unidose, etc. 
- doctor : en anglais, titre que l'on accorde non seulement aux médecins mais aussi à tout détenteur d'un Ph.D. En français, seuls les médecins ont droit au titre de docteur, les autres prennent Monsieur, Madame ou Mademoiselle avant le nom, et la mention de leur diplôme à la fin.

- $\quad$ dosage: ce terme, qui désigne non seulement le nombre de doses journalières, mais aussi leur espacement et la durée de leur administration, se rend par posologie en français. Quant au terme français dosage, il existe bel et bien mais signifie généralement la détermination exacte de la quantité d'une substance dans un milieu ou dans un mélange donné et pourrait correspondre dans certains cas au terme anglais titration ou assay.

- dramatic: faux ami dangereux, surtout dans le contexte médical. Le rendre par *dramatique qui, en français, a un sens chargé de pathos serait commettre une erreur grave. Exemple: «This treatment brought a dramatic change in the patient's condition. » Si le traitement s'est traduit par un changement très avantageux dans l'état du malade, et que I'on traduise la phrase par : «*Ce traitement a provoqué un changement dramatique dans l'état du malade», on commet un contresens grave. On peut donc rendre dramatic par spectaculaire, remarquable, notoire, etc.

- drastic: terme assez curieux, condamné dans la langue courante comme un anglicisme et pourtant accepté dans la langue médicale, mais seulement dans un cas bien particulier : quand il qualifie un remède ayant une action purgative énergique. Dans le cas de tout autre traitement ou mesure thérapeutique, il est préférable d'utiliser énergique ou draconien.

- $\quad$ early et late: danger encore, avertit Van Hoof (1970: 102). Le piège ici réside dans la polysémie anglaise des termes. Ceux-ci en effet peuvent désigner différentes choses. Ainsi, early peut signifier précoce. Exemple:

Early diagnosis is essential in carcinoma of the breast qui se traduirait par:

Un diagnostic précoce aidera à guérir cette maladie; mais il peut signifier aussi premiers ou immédiats comme dans la phrase anglaise:

The early results of the operation were good qui se lirait en français:

Les premiers résultats ou les résultats immédiats ont été satisfaisants.

M êmes observations pour late qui peut se rendre par tardif:

The late complications of measles are...

soit en français:

Les complications tardives de la rougeole sont...

$M$ ais late peut aussi signifier à long terme et la phrase anglaise:

The late results of heart transplantation are unpredictable pourrait se rendre par:

Les résultats à long terme de la transplantation cardiaque sont imprévisibles.

- fluid: n'est pas *fluide en français, mais bien liquide.

- $\quad$ incidence: ce terme est utilisé souvent à tort en anglais pour désigner la fréquence d'apparition d'un événement, sans indication de son taux d'occurrence ou de la période d'observation couverte. Dans ce cas, il s'agit d'un générique et son équivalent français serait fréquence. Ainsi, on parlera de la fréquence des effets indésirables de tel médi- 
cament. Par contre, il se traduira par incidence lorsqu'il désigne le nombre de nouveaux cas d'une maladie observés à partir d'une date donnée, par opposition à prévalence qui est le nombre absolu de cas d'une maladie à partir d'une date donnée, sans distinction entre les nouveaux cas et les cas anciens. En somme, il faudrait éviter d'utiliser *incidence pour traduire le terme anglais incidence lorsque celui-ci est utilisé comme un générique.

- $\quad$ history: dans la plupart des cas, ne se traduit pas en français par *histoire. Le terme anglais a en fait plusieurs équivalents français, qui varient selon le déterminant. Utilisé seul en anglais, history indique général ement l'ensemble des données recueillies auprès du patient, qui permettront au médecin de se faire une idée de l'état de ce patient. C'est donc son dossier médical ou son anamnèse. Mais l'on a aussi personal history = antécé dents personnels (tout événement antérieur à une maladie, qui concerne l'état de santé du sujet examiné) ; family history = antécédents familiaux (tout événement antérieur qui concerne l'état de santé de la famille du sujet examiné) ; case history = observation médicale ou histoire de la maladie (observations concernant l'évolution de la maladie, le résultat des épreuves biologiques et radiographiques, etc.). À noter que l'expression case history est souvent utilisée dans le sens d'anamnèse.

- induce: dans le sens de commencer quelque chose, to induce n'est pas *induire en français. En effet, dans cette langue, induire peut signifier tromper, comme dans l'expression «induire quelqu'un en erreur »; ou, en didactique, trouver par induction ; ou encore, uniquement dans la sous-langue de l'électricité sous la forme substantive, transmettre à distance de l'énergie électrique ou magnétique. En français médical, to induce pourrait se traduire, selon le cas, par amorcer (un traitement), provoquer (une réaction), déclencher (des effets en cascade), etc.

- $\quad$ invasive et non invasive: adjectifs anglais qualifiant une technique qui rompt, ou ne rompt pas, la continuité des tissus. II est évident qu'il ne s'agit pas d'une technique *envahissante ou *non envahissante. Devant la difficulté de traduire cette expression, on a vu apparaître dans certains écrits les qual ificatifs «français» *invasive et *non invasive; finiront-ils par avoir droit de cité ou seront-ils toujours consi dérés comme des barbarismes? Seul l'avenir nous le dira. Plusieurs équivalents ont déjà été utilisés pour en rendre le sens, dont les trop évocateurs sanglant et non sanglant, ou les imprécis traumatique et atraumatique. Finalement, J.C. Sournia (Manuila et al. 1982: 72) a proposé des termes qui, nous l'espérons, feront florès: effractif et non effractif. Ces adjectifs, dérivés d'effraction, ont le mérite d'être à la fois français et descriptifs.

- $\quad$ literature: les puristes tiquent encore devant *littérature employé en français pour désigner des publications ou de la documentation. Le mot pourtant, dans la mesure où il désigne un grand nombre d'écrits portant sur un même sujet, a tendance à s'implanter.

- $\quad$ malpractice: se traduit en français par faute professionnelle.

- $\quad$ management ( of a patient, of a disease) : il est évident qu'il ne s'agit pas de l'administration du malade ou de sa gestion, mais bien de son traitement.

- $\quad$ murmur (cardiac-) : il faut croire que le cœur ne murmure pas de la même façon en français et en anglais, puisque murmur en anglais se dit souffle en français.

- occupational : encore un terme dont l'équivalent français change selon le déterminé cette fois. Ainsi, on a:

- occupational therapy: ergothérapie ou thérapie occupationnelle;

- occupational disease: maladie professionnelle;

- occupational injury : accident du travail. 
- $\quad$ patient : grande controverse! Certains font la distinction entre patient - personne en attente d'une intervention chirurgicale ou d'un traitement - et malade - toute personne atteinte d'une maladie. Cette distinction ténue a néanmoins tendance à disparaitre.

- perfusion: terme traitre s'il en est, souvent rendu par un faux sens en français. En anglais, perfusion désigne l'irrigation - en général sanguine - d'un organe ou d'un tissu. En français, cette notion est donc rendue par irrigation sanguine. Cependant, le terme perfusion existe en français mais il désigne l'injection intraveineuse lente et prolongée d'un soluté et, dans ce sens, il est rendu en anglais par infusion.

- physician : en français, il s'agit d'un médecin. Par contre, le physicien en français est un spécialiste des sciences physiques.

- $\quad$ prescription : en anglais, le mot désignel'ordonnance, autrement dit le papier sur lequel le médecin écrit le nom et la posologie du médicament qu'il prescrit, alors que prescription en français désigne tout ce que le médecin recommande au malade en ce qui a trait à son traitement (exemple: médicament, mais aussi exercices, régime alimentaire spécial, etc.).

- rehabilitation: ce serait une erreur que de rendre ce terme par * réhabilitation, car en français il a une signification morale et sociale qui ne peut s'appliquer au contexte de la médecine. On réhabilite quelqu'un dont la réputation a été ternie ou le comportement social jugé délinquant. Par contre, on réadapte ou on rééduque un malade handicapé. Le terme anglais se rend donc par réadaptation ou rééducation.

- to refer (referring, referral) : terme assez compliqué à traduire puisqu'on ne peut le rendre par *référer (un patient à un spécialiste par exemple). En français, ce verbe se conjugue soit à la forme pronominale: se référer à quelqu'un (recourir à lui comme à une autorité) ou se référer à quelque chose (le prendre comme référence) ; soit comme verbe transitif indirect: en référer à quelqu'un (lui soumettre le cas pour qu'il en décide). To refer a patient to a specialist ne se traduit donc pas par *référer un malade à un spécialiste, mais plutôt par l'adresser à ou encore le diriger vers un spécialiste. Mais la chose se complique lorsqu'il s'agit de traduire le substantif referral. On ne peut dire I'*adresse du malade à ou sa *direction vers un spécialiste. À défaut, on peut opter, à reculons à cause de la connotation réifiante du terme, pour acheminement du malade, ou al ors se résigner à recourir à la périphrase lourde: le fait d'adresser le patient..., etc.

- route: dans le langage de la thérapeutique, c'est la voie par laquelle le médicament chemine ou est administré. Ce terme se traduit en français, selon le cas, par voie ou mode d'administration.

- $\quad$ routine: en français, la routine signifie une habitude de faire les choses toujours de la même manière. Le terme a la connotation, légèrement dépréciative, de quelque chose de machinal que l'on fait sans y penser; à la limite, de quelque chose d'ennuyeux. On ne peut donc pas traduire a routine check up par un *examen de routine, encore moins par *un examen routinier. L'équivalent serait un bilan de santé ou un examen systématique. Routine laboratory tests se traduirait par les épreuves de laboratoires habituelles ou courantes.

side effect: jusqu'à tout récemment, le calque *effet secondaire n'était entaché d'aucun blâme. Cependant certains ont fini par attacher le grelot. Ce que l'anglais désigne par side effect est en fait un effet secondaire non voulu qui, pour le moins, incommode le malade, mais qu'il doit subir pour bénéficier de l'effet thérapeutique. Dire de cet effet non désiré qu'il est secondaire n'exprime pas vraiment toute la réalité. Au terme «secondaire» n'est attaché en fait aucun sens péjoratif. Autrement dit, on peut avoir un effet secondaire à l'effet primordial recherché par le traitement mais qui serait néanmoins bénéfique. Prenons le cas de l'aspirine par exemple: administrée contre les maux 
de tête, elle pourrait avoir, comme effet secondaire, désirable, pour certains, de liquéfier le sang. Mais elle pourrait aussi avoir pour effet secondaire indésirable d'aggraver un ulcère d'estomac. Donc «secondaire» en soi n'indique rien de mauvais et pèche par son imprécision. Effet secondaire indésirable ou effet indésirable tout court aurait l'avantage d'être monosémique et beaucoup plus descriptif de la réalité.

- $\quad$ severe: n'est pas * sévère en français, mais bien grave. La résistance des auteurs francophones à renoncer à «sévère» est grande au point que cet anglicisme semble indélogeable. II n'apporte cependant rien de plus que grave, par ailleurs plus expressif.

- $\quad$ systemic: souvent rendu à tort par *systémique; encore un calque qui a la vie dure! Lorsqu'on parle de *circulation systémique, de quoi parle-t-on au juste? S'agit-il de la circulation dans un système, le système osseux par exemple, ce qui justifierait l'emploi de «systémique»? N on, on parle de circulation générale, dite encore grande circulation, par opposition à la circulation pulmonaire ou petite circulation. II s'agit donc en réal ité du cal que d'un mauvais terme anglais. M ême réflexion pour systemic symptoms. II ne s'agit pas en général de symptômes qui affectent un système en particulier mais bien de symptômes généraux, comme la fatigue, la fièvre, etc.

- $\quad$ tender : se méfier, comme le signale encore Van Hoof (1970: 103) de ce terme qui peut jouer de mauvais tours. Exemple:

The lymph glands were enlarged, firm and tender.

Que I'on traduise cette phrase par :

*Les glandes lymphatiques étaient enflées, fermes et tendres

et l'on aura écrit un faux sens et une contradiction.

Autre exemple:

There was moderate distention of the abdomen and tenderness in the periumbilical region.

Comment va-t-on traduire cette phrase?

On a constaté une distension modérée dans l'abdomen et de la *tendreté?, de la *tendresse? dans la région périombilicale?

II est évident que, dans les deux cas, il s'agit de douleur à la pression ou de sensibilité.

Voilà donc quelques exemples de faux amis contre lesquels le traducteur doit être prévenu s'il veut éviter le faux sens. Et cette liste est loin d'être exhaustive; on peut la compléter grâce au remarquable ouvrage intitulé: Dictionnaire des difficultés du français médical (Quérin : 1998).

b) Les pièges qui découlent du phénomène de la juxtaposition propre à la syntaxe anglaise

Parmi les difficultés du décodage, le traducteur devra repérer celles qui se rapportent au découpage des phrases anglaises. L'anglais en effet est une langue qui s'accommode parfaitement de la juxtaposition des différents éléments de la phrase et l'auteur anglophone ne se sent pas obligé de préciser, dans les séquences déterminants/déterminés, qui fait quoi. II peut lui arriver d'aligner les composants de la phrase, sans les mots-liens qui expliqueraient leur relation, et de compter sur l'intelligence ou l'intuition du lecteur pour faire le reste. Qui n'a lu, dans le Time M agazine, des juxtapositions de 4 ou 5 termes comprenant des adjectifs et des substantifs dans lesquels il n'est pas toujours facile de démêler les fonctions de chaque élément de la phrase. Jastrab de Saint-Robert en présente un exemple caricatural (1987: 262) : 
Earth resources remote sensing satellite data collection plaform qu'elle traduit en français par:

\section{Plate-forme de collecte de données recueillies par un satellite de télédétection des ressources terrestres.}

L'anglais médical n'est pas en reste; ainsi, on peut avoir :

Partially purified beta-lactamase preparations

Pour qui ne sait pas exactement de quoi il ressort - d'où encore une fois l'importance de la démarche documentaire - le syntagme n'est pas clair. Qu'est-ce qui est purifié: les préparations - auquel cas le participe passé se mettrait au féminin pluriel - ou la bêta-lactamase - et alors le participe passé prendrait la marque du féminin singulier?

Il y a plus grave encore: il arrive que, dans une phrase, à cause de ce phénomène de juxtaposition et donc de l'absence de certains mots clés nécessaires à la relation syntactico-sémantique qui régit les éléments de la phrase, seule une prescience de la signification de l'ensemble permet de décoder correctement les composantes de la phrase. Prenons l'exemple d'un article portant sur la résistance des antibiotiques intitulé:

The Precise Role of the Antibiotic Resistance to Beta-lactamase Hydrolysis. Que l'on traduise innocemment ce titre par:

*Le rôle précis de la résistance de l'antibiotique à I'hydrolyse de la bêta-lactamase et l'on aura commis un contresens patent, puisqu'en réalité, dans l'article, il ne s'agit nullement de la résistance de l'antibiotique à l'hydrolyse de la bêta-lactamase, mais plutôt de la résistance de l'antibiotique à sa propre hydrolyse par la bêta-lactamase. En effet, cette substance est l'agent destructeur de l'antibiotique; pour celui-ci, résister à la lyse (c'est-à-dire à la destruction) de son ennemi, serait signer son propre arrêt de mort. En réalité, pour le scientifique anglophone plus soucieux de rigueur syntaxique et de clarté, le titre anglais aurait dû être: The Precise Role of the Antibiotic Resistance to Its H ydrolysis by the Beta-lactamase et donc, en français, se lire:

Le rôle précis de la résistance de l'antibiotique à son hydrolyse par la bêta-lactamase.

c) Problèmes dus à la «mise en facteur » d'un adjectif ou d'un déterminant du nom

II existe une autre difficulté qui découle de la structure de la syntaxe anglaise: du fait qu'en anglais l'adjectif ou le déterminant du nom, qui peut se rapporter à deux ou à plusieurs noms, est placé devant ces noms, on ne sait plus, au moment du décodage, s'il se rapporte seulement au nom qui le suit immédiatement ou également à celui ou à ceux qui sont plus éloignés. Exemple, dans la phrase:

The drug's side effects are mild azotemia and proteinuria mild qualifie-t-il azotemia seulement ou également proteinuria?

Ce type de difficulté peut prendre une forme plus pernicieuse et le risque est grand, pour le traducteur qui ne comprend pas très clairement ce dont il s'agit, de mal découper la phrase et donc d'aboutir à un faux sens, voire à un non-sens. Exemple:

Gout is caused by a disturbance in purine and uric acid metabolism. 
Pour celui qui n'a de l'anglais qu'une connaissance passive - c'est le cas pour nombre de traducteurs - et qui, de plus, ne connaît pas l'étiologie de la goutte, le danger du faux sens est réel. Si, comme on pourrait être tenté de le faire, on traduit cette phrase par:

* La goutte est causée par un trouble des purines et par le métabolisme de l'acide urique on se trouve dans l'erreur à cause d'un mauvais découpage. II faut comprendre que disturbance s'applique à metabolism et qu'à son tour metabolism est déterminé par purine et par uric acid. On pourrait alors traduire la phrase par :

La goutte est causée par un trouble du métabolisme des purines et de l'acide urique. À ceux-là qui reprochent au français d'avoir besoin de plus de mots que l'anglais pour exprimer une réalité, nous faisons la preuve que l'économie dans ce domaine se réalise souvent aux dépens de la clarté.

\section{La recherche terminologique}

Le traducteur ayant dégagé le sens général du texte s'attelle alors à la recherche des équivalents des termes techniques qui lui semblent «opaques». Cette recherche peut être simple, quand les termes et leurs équivalents sont monosémiques et bien répertoriés dans les dictionnaires, ou elle peut relever du casse-tête plutôt que de la terminologie. Là, les difficultés sont de plusieurs ordres:

- l'auteur fait dans I'«à peu près». Pour désigner une notion donnée, il utilise un terme voisin de celui qu'il aurait dû employer; ou

- $\quad$ il s'agit d'un terme pour lequel le dictionnaire bilingue donne plusieurs équivalents possibles et le choix du bon ne va pas toujours de soi ; ou encore

- l'auteur est atteint de «siglomanie» aiguë et manque de la plus élémentaire politesse intellectuelle; ou enfin

- $\quad$ il s'agit d'une notion nouvelle dont le nom ne figure pas encore dans les dictionnaires, et c'est alors tout le problème de la néologie dont l'étude est trop vaste pour être abordée ici.

\section{a) Le flou terminologique}

II est imputable à la présence, dans la langue médicale, de notions voisines mais non identiques. Exemple:

mortality rate et lethality rate (ou fatality rate).

À cause de la synonymie qui existe dans la langue générale entre mortel, létal et fatal, on pourrait déduire que cette synonymie s'étend aux substantifs dont ils sont dérivés. Or, ceci n'est pas tout à fait vrai dans la langue générale et complètement faux dans la langue médicale - tant anglaise que française - puisque

- le taux de mortalité est le nombre de décès rapporté à la population générale, malades et bien-portants confondus (exemple: Le taux de mortalité annuel du sida a été de 50/ $100000)$;

alors que

- le taux de létalité est le nombre de décès rapporté à la population malade seulement (exemple: Le taux de létalité annuel du sida a été de 500/1 000).

Traduites en chiffres, ces équations révèlent de façon indiscutable l'absence de synonymie. 
Un autre exemple: trial et study. Très souvent, on a pu voir les deux termes utilisés indifféremment, parfois dans un même paragraphe, pour désigner la même notion! Or, ils ne sont pas synonymes puisque l'essai (trial) est une expérimentation à visée thérapeutique, alors que l'étude (study) est une activité d'observation, comme l'étude prospective par exemple. Et même lorsque l'étude est expérimentale, elle ne peut être confondue avec l'essai, parce qu'elle n'a pas toujours une visée thérapeutique et que, par conséquent, elle peut avoir pour objet des animaux ou même des micro-organismes, alors que l'essai ne s'applique en général qu'à l'humain.

b) Le choix du bon équivalent

Le dictionnaire bilingue donne, pour un terme anglais, un équivalent français mais cet équivalent ne semble pas s'insérer logiquement dans le contexte. Ou encore, le dictionnaire bilingue donne, pour un terme anglais, plusieurs équivalents français mais le contexte ne permet pas de privilégier l'un d'eux avec une certitude absolue. II faut alors recourir à la consultation de dictionnaires unilingues ou, à défaut, de manuels ou de revues unilingues, y trouver des contextes définitoires et ensuite juxtaposer les champs sémantiques des termes anglais et français pour s'assurer de leur équivalence absolue.

\section{c) La siglomanie}

C'est une maladie dont sont atteints bon nombre d'auteurs. II n'y aurait rien à redire à la présence des sigles, bien au contraire, s'ils étaient tous transparents. Devant certains syntagmes un peu longs, comme hypertriglycéridémie familiale, qui peuvent devenir une véritable épreuve pour qui doit les dire ou même les écrire à plusieurs reprises, il est légitime de recourir au sigle pour se faciliter la vie et économiser du temps. Cependant, la moindre des politesses exige que l'on utilise d'abord le terme entier, qu'on le fasse suivre du sigle par lequel on entend le représenter par la suite et après, mais seulement après, que l'on utilise le sigle seul. Or, ce n'est pas toujours ce qui se produit et le malheureux traducteur doit souvent jouer aux devinettes, encore heureux s'il arrive à retracer dans le texte l'expression à laquelle renvoie le sigle. Sinon, il n'a qu'un seul recours, appeler l'auteur - solution souvent coûteuse et pas toujours accessible - pour se faire expliquer la charade. Quelques ouvrages et des sections de certains dictionnaires donnent bien une liste de sigles accompagnés de leur signification, mais il arrive souvent que le terme recherché ne s'y trouve pas, soit parce qu'il est tout nouveau et n'a pas été encore attesté par les dictionnaires, soit parce qu'il est tout simplement né du cerveau de l'auteur et de son désir irrépressible d'économie.

\section{d) La néologie}

C'est là un des problèmes les plus aigus de la terminologie médicale. Les découvertes dans les domaines scientifiques - celui des sciences de la santé notamment - ont été nombreuses et importantes ces dernières années. Or, aux notions nouvelles, il faut souvent des mots nouveaux ou encore un réaménagement de ceux qui existent déjà dans la langue. M ais qu'arrive t-il lorsque cette «intendance terminologique» ne suit pas? II se creuse un vide terminologique que le chercheur sera souvent tenté de combler à sa façon, à partir de son propre réservoir linguistique - qui n'est pas 
nécessairement celui du voisin - et à partir de son propre imaginaire. II peut alors s'ensuivre une pléthore de pseudo-synonymes ou des néologismes fantaisistes qui ne s'insèrent pas toujours avec le même bonheur dans la langue médicale.

Cependant, encore une fois, le sujet est trop complexe et trop touffu pour être traité dans un paragraphe. II mérite une étude approfondie et la conjonction de plusieurs compétences.

Voilà donc, très brièvement, les différentes composantes de l'opération décodage (démarche documentaire, repérage des pièges sémantiques et recherche terminologique), ainsi que certaines des difficultés que cette phase peut présenter.

\section{B. LA PHASE DU TRANSCODAGE}

Nous aborderons maintenant la phase de transcodage. Le traducteur a donc bien compris le sens du texte, connaît les équivalents des termes «techniques» et s'apprête à rendre ce texte le plus fidèlement possible en français. Si, dans la phase de décodage, la première question que se posait le traducteur était «de quoi s'agit-il?», dans la phase de transcodage, les deux questions primordiales seront:

1. à qui le texte est-il destiné?; et

2. à quel type discursif appartient-il?

\section{Le destinataire du texte d'arrivée ou le choix du niveau de langue}

Selon que le texte s'adresse au grand public (exemple: la brochure que l'on trouve à la disposition du patient dans le cabinet du médecin), ou au personnel paramédical (manuel de formation ou argumentaire destiné aux visiteurs médicaux), ou encore au médecin spécialiste ou généraliste (article scientifique ou films dans le cadre de la formation continue), le traducteur choisira, dans la palette des niveaux de langue dont il dispose, celui qu'il utilisera pour transcoder le texte de départ. II est évident que, s'il s'agit d'une brochure grand public, parler d'érythème au lieu de rougeur, d'hyperlipidémie au lieu d'un excès de gras dans le sang ne semble pas très astucieux. Inversement, dans une monographie destinée à informer le médecin sur les caractéristiques d'un produit donné, ou encore dans un tiré à part d'une revue scientifique, on ne pourra pas parler de *maux de tête ou de *démangeaisons; on choisira un niveau de langue plus jargonneux, plus savant, et l'on parlera de céphalée et de prurit, même si, ce faisant, on diffère du niveau de langue adopté par l'anglais. Celui-ci, en effet, ne s'embarrasse guère de ces distinctions et utilisera volontiers, quel que soit le destinataire du texte, le mot de la langue générale. C'est ainsi que, dans des textes très spécialisés, on trouvera fréquemment headache, backache, joint pain, rash, etc.

\section{Le type discursif du texte à traduire}

La «tonalité» de l'écriture - le choix du style et celui des mots selon leur connotation - seront fonction du type du texte à traduire. Celui-ci, en effet, dans le contexte de la traduction médicale, peut être:

- informatif, comme la monographie du produit ou l'article scientifique. II a alors pour unique but de transmettre le plus fidèlement possible l'information. Il commandera des mots neutres, univoques, sans connotation péjorative ou méliorative, et un style économique et concis. 
- $\quad$ exhortatif, comme la pièce publicitaire, celle qui présente au médecin les avantages potentiels d'un médicament. Ici, le texte - comme tout texte publicitaire - ne se contentera pas de transmettre une information, mais il s'efforcera de provoquer chez le lecteur ou l'auditeur une réaction psychologique qui se traduirait par un acte, en l'occurrence la prescription de tel médicament, de préférence à tel autre. Les moyens stylistiques utilisés par le texte de départ pour influencer le destinataire devront al ors trouver leur pendant dans la langue d'arrivée. Ce type de texte fera appel à la compétence publicitaire du traducteur, donc à ses connaissances des nuances linguistiques et stylistiques, mais surtout à sa créativité; celle-ci cependant devra être contenue dans les limites, très étroites, imposées par les principes d'éthique et de prudence inhérents à tout ce qui touche à la santé de l'humain.

Mentionnons enfin que le texte peut être de type expressif, et là le traducteur s'attachera à rendre essentiellement, et avec la plus grande fidélité, les émotions de I'auteur. C'est, par exemple, le cas du poème. Il est évident que ce troisième type de texte trouvera rarement sa place dans la traduction médicale.

\section{Le repérage des pièges propres à la phase de transcodage}

Une fois que le traducteur aura choisi le niveau de langue et le style du texte à transcoder, il lui restera à éviter certains pièges qui peuvent se creuser sous ses pas à cette étape du transcodage. En voici brièvement quelques-uns:

a) Les faux amis morphologiques

Ils sont plus bénins que les faux amis sémantiques, à condition que l'on prenne I'habitude de vérifier l'orthographe devant le moindre doute. Ainsi, le traducteur pressé - que l'on nous pardonne cette redondance - qui ne vérifie pas l'orthographe d'exsudat risque d'omettre l's qui n'existe pas dans le mot anglais. Ou encore, sous l'influence de la langue de départ, il risque d'écrire agressif avec deux $\mathbf{g}$. II faudra aussi qu'il se souvienne que l'équivalent français de hemorrhage perd le $\mathbf{h}$ du milieu, que edema s'écrit œdème en français, etc.

b) Le problème de l'adjectivation

Certains termes anglais ou français peuvent subir l'adjectivation sans problème. Exemple: resistance of a bacteria devient bacterial resistance, comme son pendant français résistance d'une bactérie peut s'écrire résistance bactérienne. Cependant, ce modèle ne s'applique pas avec un parallélisme constant à l'anglais et au français. Les cas cités par Van Hoof (1970: 99-100) sont les paires culture/culture et skeleton/ squelette. On rencontre souvent en anglais l'expression cultural results. II est évident qu'il ne viendrait à l'esprit de personne de la traduire en français par *résultats culturels, mais bien par résultats des cultures. Également, certains hésiteraient à traduire skeletal muscles par *muscles squelettiques, à cause del'aspect affectif de l'expression et lui préféreront muscles du squelette. C'est d'ailleurs cette appellation que M anuila choisira comme vedette dans son dictionnaire (Manuila et al. 1972: 893).

\section{c) Le problème des désinences}

Comme on le sait, la désinence est cet élément variable qui s'ajoute au radical pour modifier la catégorie grammaticale. II peut arriver que, pour un même déterminant 
anglais, le français en ait deux dont la désinence varie selon la nature du déterminé. Exemple, le mot coronary aura deux désinences différentes en français: coronaire et coronarien, ou encore suprarenal qui peut être surrénal ou surrénalien. Aucune règle n'est donnée pour le choix de l'un ou l'autre adjectif. Une hypothèse cependant: la première forme s'applique à une entité anatomique et la seconde à ce qui se rapporte à cette entité. C'est ainsi qu'on dira: des artères coronaires, mais des troubles coronariens. Ou encore des glandes surrénales, mais une tumeur surrénalienne.

Par ailleurs, il existe un autre problème concernant les désinences: I'absence de tout modèle constant pour le passage d'une désinence anglaise à son pendant français. Prenons la terminaison en «al » par exemple. On a nasal = nasal, vaginal = vaginal, renal = renal, mais gare à celui qui croit à l'universalité de ce modèle, car on a aussi bacterial = bactérien, placental = placentaire, streptococcal = streptococcique, etc. C'est là que la démarche documentaire, de par les lectures qu'elle impose, paie des dividendes: le traducteur intègre le bon vocabulaire, pressent les pièges et évite ainsi d'y tomber.

d) Le problème de la voix passive et de la forme gérondive ou les pièges de l'anacoluthe

À quoi faut-il imputer ce problème structurel ? À la modestie des auteurs anglophones ou à leur excessive prudence? Toujours est-il qu'ils sont très souvent absents de leurs recherches ou des résultats de celles-ci. Exemple:

$M$ ice were injected with propranolol to determine its side effects.

II s'agit là d'un problème qui donne lieu assez souvent à une entorse à la syntaxe française, entorse qui porte le joli nom d'anacoluthe (du grec anacoluthon qui signifie «absence de suite»). En fait, il s'agit d'une absence de suite, d'une rupture, dans la construction logique d'une phrase. Que l'on nous permette, pour nous expliquer, de revenir à la bonne analyse logique d'antan. Chaque proposition a un verbe et un sujet. Lorsqu'une proposition subordonnée n'a pas un sujet exprimé, on présume qu'elle est gouvernée par le sujet de la proposition principale. C'est ainsi que, dans la phrase:

Pierre tomba en montant les escaliers ou

En montant les escaliers, Pierre tomba

Pierre est le sujet des deux propositions, comme il se doit. Or, dans l'anacoluthe, c'est cette structure logique qui se trouve rompue. Nous donnerons, pour illustrer ce phénomène de déstructuration syntaxique, quelques exemples pris d'abord dans la langue générale, puis dans la langue médicale.

Dans la phrase:

En montant les escaliers, la valise tomba des mains de Pierre

le sujet de la proposition gérondive n'étant pas celui de la proposition principale, on a une anacoluthe évidente.

Plus évidente encore, l'anacoluthe l'est dans la phrase qui suit:

En traversant la rue à bicyclette, une vache le renversa.

Syntaxiquement, le sujet de la subordonnée gérondive étant le même que celui de la principale... on se retrouve avec une vache cycliste! L'on nous dira que le sens est clair. Soit, mais le français est plus exigeant sur ce chapitre et nous connaissons des 
cas de refus de candidature qui sont dus à la formule de salutation de la demande d'emploi, qui se lisait ainsi :

En attendant votre réponse, veuillez agréer, M onsieur, l'assurance de mes sentiments les meilleurs.

Là aussi, l'erreur est la même: le participe présent placé au début de la phrase n'a pas en réalité le même sujet que le verbe de la proposition principale. Ce n'est pas le Monsieur qui attend la réponse, c'est le candidat... et elle risque d'être négative à cause de la construction fautive de la phrase.

La même règle s'applique lorsque la proposition subordonnée est une proposition infinitive:

J'ai donné mon manuscrit à un ami afin de l'évaluer.

Si l'auteur veut évaluer son ami, il n'y a rien à redire, mais si l'ami doit évaluer le manuscrit, alors la phrase est fautive.

Relevé, par ailleurs, dans un grand quotidien montréalais:

Pour avoir entraîné un millier de mineurs à occuper durant 12 jours la mine de Ziemovit, le tribunal militaire de Katowice a prononcé neuf peines allant de 3 à 7 ans de prison.

Ou encore, dans un journal étudiant, à propos de Raymond Aron:

M ort le 17 octobre dernier, le monde entier (vous parlez d'une hécatombe!) s'aperçoit de

l'ampleur de sa contribution à l'explication des sociétés de l'Ouest et de l'Est.

On pourrait nous rétorquer qu'il ne s'agit pas là d'écrits scientifiques. II reste que, sous l'influence des auteurs anglophones, le traducteur peut être tenté, par mimé tisme, de reproduire le même modèle syntaxique. Avec pour résultat des phrases du genre:

Les patients doivent faire l'objet d'une étroite surveillance médicale afin de déceler le début de l'épisode dépressif.

Si les patients font I'objet d'une étroite surveillance, ce ne sont pas eux qui décèleront le début de l'épisode dépressif !

Un autre exemple:

Les patients traités pendant plus de 24 mois sont en nombre insuffisant pour conclure définitivement à un effet protecteur persistant après le $24^{\mathrm{e}}$ mois.

À l'évidence, ce ne sont pas les patients qui concluent ou ne concluent pas, mais bien les chercheurs. Ou mieux encore:

Un patient qui présente des symptômes de démence subit généralement un examen neurologique approfondi en vue de déceler des affections du système nerveux pouvant contribuer à la manifestation de ces symptômes.

Est-ce bien le patient atteint de démence qui est capable de déceler les affections du système nerveux?

Lu par ailleurs, dans la très respectable Encyclopédie médico-chirurgicale (Cahier 8065 F10):

Les transfusions de sang ont joué un rôle très important, mais il a notablement diminué, d'une part en réduisant le nombre de donneurs de sang [...] et en décelant par des méthodes sensibles la présence de l'Ag H BS (I'antigène de l'hépatite).

Qui a réduit le nombre de donneurs de sang ?... et qui a décelé la présence de l'antigène? L'acteur réel est totalement absent de cet énoncé pour le moins échevelé! 
Quel recours a le traducteur pour traduire correctement la phrase anglaise qui, abusant de la voix passive ou de la forme gérondive, tombe dans le piège de l'anacoluthe? Quelques solutions sont possibles:

- Rétablir le ou les véritables sujets de l'action exprimée par la proposition subordonnée, en parlant des chercheurs ou des expérimentateurs. Ainsi, pour reprendre l'un des exemples précédents, on aurait:

Les chercheurs ont surveillé étroitement l'état des malades afin de déceler...

ou

- Utiliser le «nous», si ce pronom apparaît dans la suite de l'article:

Nous avons surveillé étroitement l'état des malades afin de déceler...

ou encore

- Redonner, à l'aide du pronom passe-partout «on», un sujet distinct à la proposition infinitive:

Les patients traités pendant plus de 24 mois sont en nombre insuffisant pour que l'on puisse conclure à un effet protecteur...

e) Les problèmes de synonymie

Le langage médical est peut-être le plus beau cas de prolifération synonymique que I'on puisse imaginer. II s'agit là d'une véritable orgie. Et cela vaut pour les deux langues. En anglais comme en français, la même entité pathologique peut être désignée par une dizaine de termes.

Ainsi, en anglais, angioneurotic edema, une variété d'urticaire caractérisée par la formation brusque d'infiltrations œdémateuses sous-cutanées, a de nombreux synonymes: acute circumscribed edema, acute essential edema, angioedema, Bannister's disease, giant urticaria, migratory edema, M ilton's disease, M ilton urticaria, nonhere ditary angioneurotic edema, Q uincke's disease, Quincke's edema, etc.

Et cette synonymite aiguë envahit également le français où l'on a en effet, pour la même maladie, œdème de Quincke, maladie de Quincke, maladie de Milton, urticaire géante, angioneurose cutanée, œdème aigu angioneurotique, urticaire œdémateuse, etc.

II y a certainement là un danger, celui de la «babélisation» du langage. Cette prolifération synonymique malsaine explique que les usagers de la langue médicale se soient émus et aient entrepris une opération de normalisation, qui a commencé avec la rédaction du Dictionnaire français de médecine et de biologie et qui se poursuit toujours dans les sous-langues des spécialités médicales, jeunes ou rajeunies, dont le vocabulaire ne s'est pas encore stabilisé. La démarche de l'équipe qui a conçu ce dictionnaire en trois volumes et qui est exposée dans l'introduction a été la suivante:

1. choisir le terme ayant reçu l'aval des spécialistes du domaine et dont la fréquence d'utilisation est la plus grande pour en faire le terme vedette sous lequel apparaîtra la définition;

2. donner la liste des synonymes de ce terme;

3. faire figurer chaque synonyme à la place alphabétique qui lui revient, en mentionnant «synonyme de... (la vedette qui a fait l'objet de la définition)».

On peut donc déduire de cette démarche assez rigoureuse que, devant une série synonymique trouvée dans un dictionnaire d'équivalences bilingues, le choix du traducteur pourrait sans trop de risque se porter sur celui que le Manuila ou l'un des deux autres «grands» - le Flammarion ou le Garnier-Delamare - ont privilégié 
comme terme vedette. Malheureusement, les choses ne sont pas aussi simples. En effet, il arrive que ces trois dictionnaires francophones ne s'entendent pas sur le terme à placer en vedette. Par ailleurs, ces ouvrages témoignent de la fréquence de l'usage en France. Or, nos médecins du Québec, exposés sans relâche aux publications anglophones, sont tentés de privilégier le terme qui se rapproche le plus du terme anglais, parfois sciemment pour être compris des gens d'ici.

Alors que faire? On peut, dans le cas où le choix des trois dictionnaires cités plus haut se porte sur le même terme, opter sans grand risque d'erreur pour ce terme; dans le cas contraire, il faudrait procéder par sondage auprès des professionnels de la santé et choisir le terme qui emporte le plus de suffrages.

II existe encore un autre piège, plus subtil celui-là. Pour un terme anglais, il existe parfois plusieurs équivalents français qui ne sont pas tout à fait interchangeables dans la mesure où chacun d'eux accompagne un cooccurrent particulier. C'est le cas par exemple du terme anglais fold qu'on peut être tenté de traduire constamment par pli. Or on a (Van Hoof 1970: 106):

nail fold = sillon latéral de l'ongle ou sillon périunguéal ;

ciliary fold = vallées ciliaires;

palatine folds $=$ crêtes palatines, etc.

f) Les problèmes d'éponymie

La prolifération des éponymes dans toutes les branches de la médecine est presque incontrôlable. L'éponyme s'applique aux signes, aux symptômes, aux maladies, quelquefois même aux structures anatomiques. Les éponymes médicaux sont de différentes sortes:

- Le nom propre donne naissance à un nom commun identique dans les deux langues: addisonism = addisonisme.

- Lenom propre reste un nom propre, dans les deux langues: Parkinson disease $=$ maladie de Parkinson.

- Les deux termes ne sont pas de même nature en anglais et en français, I'un étant un éponyme l'autre une expression descriptive: Fallopian pregnancy (nommé d'après Fallop) devient grossesse tubulaire ou extra-utérine.

- $\quad$ Un éponyme anglais peut avoir comme équivalent un éponyme français différent, chacun portant le nom de celui qui a décrit la maladie en premier et qui peut être une personne différente dans les deux groupes linguistiques (nationalisme oblige!) ;

Exemple: Reiter's disease chez les anglophones devient syndrome de FiessingerLeroy en France.

Autre aspect du casse-tête: une même affection est désignée par plusieurs éponymes différents. Le cas type souvent cité est le goitre exophtalmique que certains appelleront maladie de Basedow, d'autres maladie de Graves, d'autres encore maladie de Mars ou maladie de Flajani.

g) Le problème des abréviations

Tout jargon scientifique ou technique qui se respecte se forge ses propres abréviations. Le langage médical n'échappe pas à la règle et cela ne facilite guère le travail du traducteur, puisqu'il va sans dire que, dans la grande majorité des cas, les abréviations ne seront pas - ou ne devraient pas être - les mêmes en français et en anglais. 
Exemple: R.A. qui est l'abréviation de rhumatoid arthritis

aura pour équivalent P.R. abréviation de polyarthrite rhumatoïde.

Certaines abréviations par bonheur ne changent pas et tout traducteur médical apprend vite à les connaître, comme:

IV = intraveineux (dans les deux langues)

IM = intramusculaire

B.K. = Bacille de Koch

Mais il arrive que la même abréviation indique deux ou plusieurs notions différentes et I'on a par exemple:

SR qui peut être l'abréviation de:

sedimentation rate $=$ vitesse de sédimentation

sinus rhythm $=$ rythme sinusal

slow-release $=$ à libération lente

stimulus response $=$ stimulus-réponse

Souvent par contre, le français reprend telles quelles les abréviations créées par l'anglais. C'est le cas de l'hormone adrénocorticotrope ou corticotrophine, abrégée en ACTH (de l'anglais adreno-cortico-tropic hormone), ou encore HDL (abréviation de l'expression anglaise high density lipoprotein). II arrive même que les auteurs francophones poussent très loin la fidélité à l'anglais, par exemple jusqu'à adopter telle quelle l'abréviation LH-RH (del'expression anglaise plutôt lourdeluteinizing hormonereleasing hormone) pour désigner... la gonadolibérine! (Jammal 1992: 213-214).

De toute manière et au risque de nous répéter, il y a une politesse à observer à l'égard du lecteur, qui consiste d'abord à écrire le terme ou la locution nominale en toutes lettres la première fois, suivie de l'abréviation entre parenthèses, quitte par la suite à n'utiliser que l'abréviation. Par exemple, on parlera d'abord de concentration minimale inhibitrice (CMI) et I'on emploiera ensuite le sigle CMI. À une exception près, lorsque le sigle est tellement connu - s'il s'agit de l'ADN par exemple - qu'il devient inutile de le mentionner en entier. Dans le cas des abréviations anglaises reprises telles quelles dans le texte français parce que leurs équivalents français ne sont pas connus ou pas encore acceptés, on fera suivre l'abréviation anglaise de la mention («de l'anglais... » avec le terme écrit en toutes lettres).

Nous n'avons mentionné jusqu'ici que les pièges les plus courants de la traduction médicale. Nous nous permettrons de rappeler très brièvement quelques autres difficultés communes à tous les domaines de traduction mais qui se présentent, peutêtre de façon plus subtile encore, dans la langue médicale.

\section{Le zeugme}

Comme on le sait, on appelle zeugme une construction qui consiste à éliminer de l'énoncé un ou plusieurs mots que l'esprit peut rétablir aisément. Exemple:

L'un prit le chapeau, l'autre le manteau.

II s'agit là d'un zeugme légitime, tout à fait correct. Cependant, cette construction syntaxique peut être traîtresse. En effet, elle devient incorrecte dès lors qu'on adjoint à deux verbes - ou à deux adjectifs qualificatifs ou à deux participes passés sans auxiliaires ou encore à deux adverbes - un seul et même complément ou la même préposition si, pris séparément, ces verbes, ces qualificatifs, ces participes n'acceptent 
pas ce complément ou cette préposition sous la même forme. Exemple pris dans la langue générale:

Il est monté et descendu de voiture en moins de temps qu'il ne faut pour le dire. Ici, le mauvais zeugme est facile à repérer. Par contre, dans l'énoncé suivant définissant, dans le contexte de l'épidémiologie, la notion de réservoir (d'agent infectieux) :

Tout être vivant ou tout milieu qui héberge et favorise, pendant une période relativement prolongée, le développement d'agents infectieux

I'accroc à la syntaxe est plus subtil. En effet, il faut se rendre compte que le milieu peut favoriser le développement mais qu'il ne peut l'héberger.

\section{Les faux comparatifs}

II s'agit là d'un piège qui se creuse souvent sous les pas de l'apprenti traducteur. Assez fréquemment, l'anglais emploie un comparatif qui n'en est pas vraiment un puisque l'autre élément de la comparaison est absent; exemple:

Older people are more at risk of Parkinson's disease

sans référence préalable à un groupe plus jeune. Traduire cela par : *Les personnes plus âgées..., sans que l'on sache plus âgées que qui, constitue pour le moins une maladresse. La plupart du temps, il ne s'agit d'aucune comparaison et older people pourrait se rendre tout simplement par les personnes âgées. Les faux comparatifs abondent dans les textes médicaux; il faut être vigilant pour ne pas y succomber.

Dans cette méthodologie propre à la traduction médicale, nous avons traité essentiellement de deux phases de l'opération traduisante - le décodage et le transcodage - parce qu'elles présentent toutes deux une démarche et des pièges particuliers à ce domaine. Mais il va sans dire qu'en traduisant, il faut tenir compte des impératifs, universels ceux-là, d'une troisième phase, celle que nous appellerons «rédactionnelle», avec tout ce qu'elle implique d'exigence de clarté, de logique, de style et d'élégance dans l'expression de la pensée. À ces impératifs, le traducteur médical ne peut se soustraire, quelles que soient la nature, l'imperfection ou la difficulté du texte à traduire. «Il faut toujours, pour bien écrire, avoir quelque chose à dire», dit Goethe. Pour le traducteur, qui a franchi l'étape du «quoi dire», il restera à se concentrer sur le «bien écrire».

\section{NOTE}

1. Nous nous référons, dans cet article, à la traduction de l'anglais au français.

\section{RÉFÉRENCES}

Dictionnaire de médecine Flammarion (1989) : Paris, Flammarion M édecine-Sciences.

Garnier, M. et V. Del amare (1992): Dictionnaire des termes techniques de médecine, Paris, $M$ aloine.

Jammal , Amal et Pierre Dussault (1983) : L'hypertension artérielle, Montréal, Sodilis.

Jammal , Amal, Geneviève Losl ier et Robert Al I ar d (1988) : Dictionnaire d'épidémiologie, SaintHyacinthe/Paris, Edisem/M aloine.

Jammal , Amal (1992) : «L'hybridation du langage médical français, un phénomène inévitable?», Le journal de radiologie, Paris, Masson, p. 213-214.

Jast r ab de Saint -Robert, M. J. (1987) : «Les syntagmes nominaux complexes en anglais et en français: éléments de réflexion», M eta, 32 (3), p. 260-266. 
Le nouveau petit Robert (1994) : Paris, Dictionnaires Le Robert.

Le petit Larousse illustré (1998) : Paris, Larousse.

M anuil a, A., L. M anuil a, M. Nicol e et H. Lambert (1972) : Dictionnaire français de médecine et de biologie, t. II, Paris, M asson.

M anuila, A., M anuila, L. et J. C. Sournia (1982) : Dictionnaire français de médecine et de biologie, 20 vol. + Supplément, Paris, Masson.

Quér in, Serge (1998) : Dictionnaire des difficultés du français médical, Saint-Hyacinthe/Paris, Edisem/Maloine.

Sch u mach er , Nestor (1973) : «Analyse du processus de traduction : conséquences méthodologiques», M eta, 18 (3), p. 308-314.

Van H o of , Henri (1970) : «La traduction médico-pharmaceutique», M eta, 15 (2), p. 95-109.

- - (1986) : Précis pratique de traduction médicale (anglais français), Paris, Maloine. 